\title{
Post-Kidney Transplant Anemia as a Result of Passenger Lymphocyte Syndrome by Rhesus Antibodies - A Rare Etiology for a Common Finding
}

\author{
Andreia Dias da Silva, ${ }^{1, *}$, Jorge Malheiro², Manuela Almeida², La Salete Martins ${ }^{2}$, Leonídio Dias², Sofia Pedroso², António Castro \\ Henriques $^{2}$ and António Cabrita²
}

${ }^{1}$ Centro Hospitalar Tondela-Viseu, Portugal

${ }^{2}$ Centro Hospitalar do Porto, Portugal

*Corresponding author: Andreia Dias da Silva, Centro Hospitalar Tondela-Viseu, Portugal, E-mail: andreiaraquelsilva89@gmail.com

Received: 26 Jul, 2019 | Accepted: 14 Aug, 2019 | Published: 21 Aug, 2019

Citation: da Silva AD, Malheiro J, Almeida M, Martins LS, Dias L, et al. (2019) Post-Kidney Transplant Anemia as a Result of Passenger Lymphocyte Syndrome by Rhesus Antibodies - A Rare Etiology for a Common Finding. Int J Nephrol Kidney Fail 5(2): dx.doi.org/10.16966/23805498.179

Copyright: (C) 2019 da Silva AD, et al. This is an open-access article distributed under the terms of the Creative Commons Attribution License, which permits unrestricted use, distribution, and reproduction in any medium, provided the original author and source are credited.

\begin{abstract}
Posttransplant Anemia (PTA) is a common finding, often multifactorial. Some atypical etiologies can be masked by the usual causes, resulting in non-solving anemia and compromising graft viability and host recovery. An immune-mediated hemolysis can develop in solid or bone marrow graft recipients in the presence of $\mathrm{ABO}$-minor mismatch or, more rarely, other blood groups mismatches, like Rhesus (Rh) system. This condition, known by Passenger Lymphocyte Syndrome (PLS), occurs after the transfer of donor's viable B-lymphocytes with the transplanted organ, which leads to the production of antibodies (Ab) against recipient red blood cells (RBC) antigens (Ag). PLS should be considered even when the PTA etiology seems obvious or when there are no apparent reasons for suspecting it. We review a case of PLS after kidney transplantation, caused by an Rh(D) mismatch.
\end{abstract}

Keywords: Hemolytic anemia; Kidney transplant; Rhesus system mismatch; Passenger lymphocyte syndrome

\section{Introduction}

Anemia is a common feature of posttransplant period and usually occurs in a multifactorial context, in particular: prior low hemoglobin $(\mathrm{Hb})$ values (lower-than-normal $\mathrm{Hb}$ targets in chronic kidney disease patients), surgical blood loss, inflammation, frequent phlebotomy, dilutional anemia secondary to aggressive perioperative volume expansion and erythropoietin and/or iron deficiency. These conditions can be exacerbated by bone marrow suppression, caused by immunosuppressive and infection prophylaxis medications, infectious events or even graft dysfunction or rejection. Nevertheless, another potential cause to be considered is hemolytic anemia, which can be microangiopathic (secondary or atypical hemolytic uremic syndrome, thrombotic thrombocytopenic purpura) or autoimmune.

A rare condition known as Passenger Lymphocyte Syndrome (PLS) results in Autoimmune Hemolytic Anemia (AIHA). PLS typically occurs after solid organ or bone marrow transplant, in cases of minor mismatch in the ABO system between the recipient and the donor [1]. Less commonly, it also occurs with other blood group mismatches like $\mathrm{Rh}$ (especially $\mathrm{D}$ ) system $[2,3]$ specifically when a $\mathrm{RhD}^{+}$recipient receives an organ from a previously isoimmunized $\mathrm{RhD}^{-}$donor. The pathophysiology of PLS relies on the concomitant transfer of donor's viable B-lymphocytes and/or plasma cells with the transplanted organ, which are reactive or stimulated to produce antibodies $(\mathrm{Ab})$ against recipient's red blood cells (RBC) antigens (Ag), causing extravascular hemolysis (although some complement-mediated hemolysis can occur as well) $[4,5]$.

The incidence of PLS has been reported as $9 \%$ for kidney, $29 \%$ for liver and $70 \%$ for heart-lung transplants [6]. Further, it is probably associated to the extent of lymphoid tissue implanted with the corresponding organ transplant. Regarding PLS following kidney transplant, there are few reported cases resulting from $\mathrm{Rh}$ mismatch $[2,6,7]$ and the majority were not associated to clinically relevant hemolysis [8].

PTA induced by PLS immune-mediated hemolysis is usually abrupt in onset, but self-limited. Analytically, hemolysis signs may be mild and, therefore, it is necessary a high degree of suspicion for its diagnosis. A positive direct antiglobulin test (DAT) is present, in most cases caused by IgG-Ab only [6]. The detection of donor's RBC Ab in a recipient's blood sample confirms the diagnosis. Blood transfusion, from the donor's blood group type, and an increased steroid dose may be necessary for the treatment of this entity [1].

Herein, we report a case of PLS associated with an Rh (D) mismatch. The same clinical case was summarily described by Antunes MB, et al. [9], with detailed reporting from a transfusion medicine and immunological viewpoint. Therefore, we found pertinent to review this case from a clinical transplantation perspective, giving the rarity of the condition and the interesting clinical course of the patient. 


\section{Case Report}

We present a case of a 53 years old female patient, with a history of end-stage renal disease secondary to IgA nephropathy undergoing hemodialysis and a previous living-donor kidney transplant 3 years ago, which was lost following renal artery thrombosis (etiology's further study was inconclusive) and nephrectomy 24 hours postprocedure.

She was admitted for a second living-donor kidney transplant in July of 2018. The donor, a 45 years old female friend, had an $\mathrm{O}$ $\mathrm{Rh}^{-}$blood group and the recipient was $\mathrm{O} \mathrm{Rh}^{+}$. Both were IgG+ for Cytomegalovirus and Epstein Barr virus. The transplant had a low immunologic risk, with negative crossmatch by flow cytometry and complement-dependent cytotoxicity, panel-reactive antibody of $0 \%$ and detection of only one antibody, anti-DQA, non-donor specific. There were 5 donor-recipient HLA mismatches. She underwent immunosuppression with basiliximab for induction and tacrolimus, mycophenolate mofetil and methylprednisolone/prednisolone as maintenance therapy.

The transplant was complicated during the vascular surgical implant (iliac artery thrombosis, submitted to thrombectomy) and in the immediate post-transplant period by hemorrhagic shock (although without active hemorrhage detected in the reintervention, just a minor subcapsular hematoma). After recovery of hemodynamic stability, a graft doppler was compatible with renal artery stenosis and therefore the patient was submitted to angiography with balloon angioplasty, a procedure that was complicated by rupture of renal artery needing surgical correction. In this 48 hours posttransplant period, values of $\mathrm{Hb}$ decreased 3,6 g/dl (pre-transplant $\mathrm{Hb}$ value of 12,5 g/dl; minimal value of $8,9 \mathrm{~g} / \mathrm{dl}$ ), and the patient was transfused with 4 units of RBC concentrate $\mathrm{O} \mathrm{Rh}^{+}$.

ThepatienthadDelayedGraftFunction(DGF), needinghemodialysis until day 5 posttransplant. At day 3 , she initiated thymoglobulin that was maintained for 7 days, as a result of subtherapeutic levels of tacrolimus associated with its enteral administration of the medication being intermittently unavailable. Graft biopsy was not performed, not only because of multiple hemorrhagic complications, but also since DGF was considered to probably be an acute tubular necrosis secondary to the prior vascular complications and hemorrhagic shock. In fact, she recovered diuresis at day 4 , with progressive improvement of her graft function and normalization of serum creatinine at day 16 $(0,9 \mathrm{mg} / \mathrm{dl})$.

Nevertheless, she maintained a normocytic normochromic anemia ( $\mathrm{Hb}$ values ranging between $6.2-8.8 \mathrm{~g} / \mathrm{dl}$ ), poorly responsive to $\mathrm{RBC}$ transfusion (she had 4 more units of $\mathrm{RBC} \mathrm{O} \mathrm{Rh}^{+}$between day 3 and day 12) and introduction of epoetin beta. The anemia was initially interpreted to be multifactorial blood losses after surgery, inflammation, thymoglobulin toxicity. At day 14, it was detected Autoimmune Hemolytic Anemia (AIHA): hemoglobin of $5 \mathrm{~g} / \mathrm{dl}$, high serum lactate dehydrogenase (365 U/L), hyperbilirubinemia by indirect bilirubin (total bilirubin of $2,38 \mathrm{mg} / \mathrm{dl}$ ), reticulocytosis $(17,39 \%)$, peripheral blood smear with markedly anisocytosis and spherocytosis, haptoglobin undetectable $(<0,24 \mathrm{~g} / \mathrm{L})$ and a positive DAT (4+). Anti-D Ab was detected, and the diagnosis of PLS immunemediated hemolysis was made. The patient immediately initiated $4 \mathrm{mg}$ intravenous dexamethasone (4 days) followed by oral prednisolone $60 \mathrm{mg} /$ day for 10 days, and then progressively reduced to $20 \mathrm{mg}$ /day. Additionally, epoetin beta was stopped and there was the need for 7 more $\mathrm{RBC}$ units from the donor's blood type ( $\left.\mathrm{ORh}^{-}\right)$, keeping values of hemoglobin between $6.9-8 \mathrm{~g} / \mathrm{dl}$. During this period, she presented symptoms of asthenia, without other clinical complains. She was discharged at day 45 clinically stabilized, with a $\mathrm{Hb}$ value of $9 \mathrm{~g} / \mathrm{dl}$, $5 \%$ of reticulocytes, solved hyperbilirubinemia and serum LDH of 282 U/L. Kidney graft function maintained stable (serum creatinine at discharge of $1,1 \mathrm{mg} / \mathrm{dl}$ ). After a six-month follow-up period, the patient maintains controlled $\mathrm{Hb}$ values (11-12 g/dl) with anti-D titers $<1 / 2$, without the need of further RBC transfusions or erythropoiesis stimulating agents and under $5 \mathrm{mg} /$ day of prednisolone. In graphic 1 we present the analytical evolution of this PTA case, as a result of PLS mediated by $\mathrm{Rh} \mathrm{Ab}$.

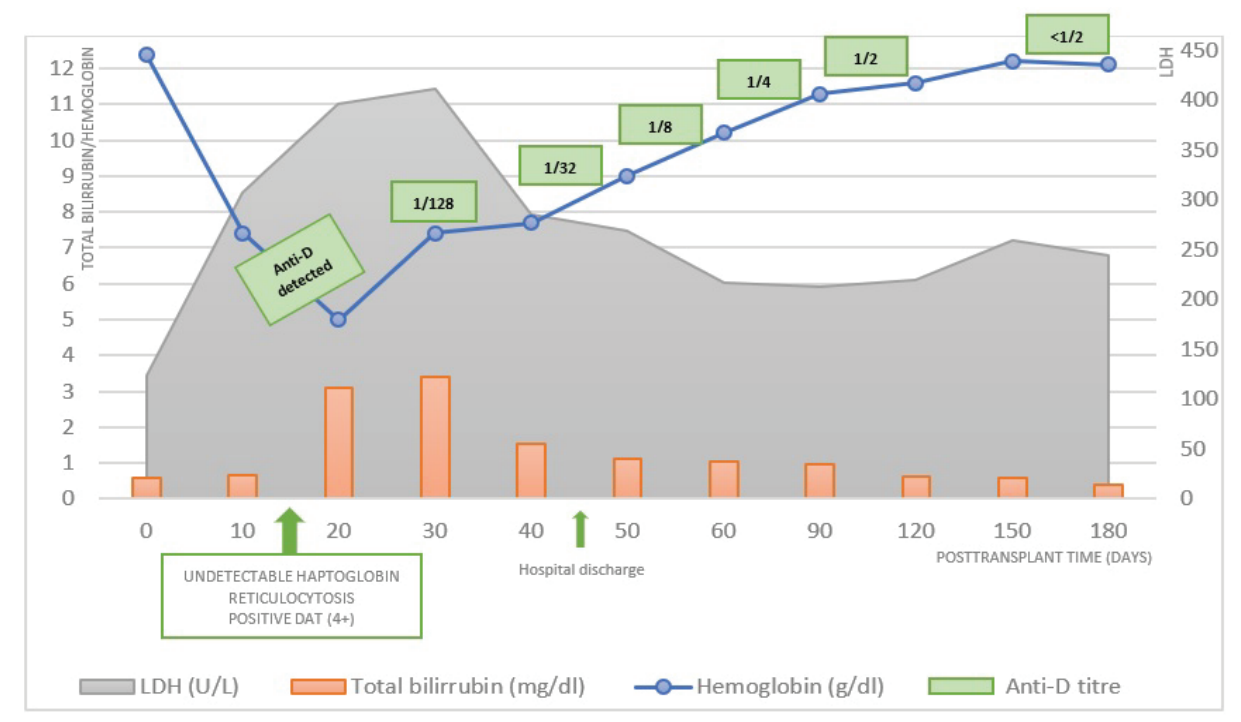

Graphic 1: Posttransplant timeline, with detailed analytical features of PLS immune-mediated hemolysis of our patient. The diagnosis was made at day 14 , and the patient immediately initiated intravenous dexamethasone (4 days) followed by oral prednisolone $60 \mathrm{mg} / \mathrm{day}$ for $10 \mathrm{days}$, and then progressively reduced to $20 \mathrm{mg} /$ day. The patient needed 7 more units of RBC concentrate, ORh-, the last one done the day before of discharge. DAT- Direct Antiglobulin Test; LDH- Lactate Dehydrogenase 
We investigated the etiology of donor's anti-D Ab and uncovered that the donor had a previous miscarriage, with a possible maternal $\mathrm{Rh}(\mathrm{D})$ alloimmunization. In fact, she had two term pregnancies afterwards, where the two babies suffered hemolytic disease of the newborn.

\section{Discussion and Conclusion}

PLS is an uncommon cause of PTA. Nevertheless, it must be considered as a possible etiology when there is a known minor $\mathrm{ABO}$ or other RBC mismatch between the donor and the recipient, or when the attempts to correct the anemia from other identifiable possible causes fail. In the reported case, the patient presented an anemia that could be justified by the initial blood loss or by ATG toxicity, but its persistence after RBC's transfusion of recipient's blood type and introduction of epoetin beta, motivated us to search for other causes.

Of notice, not all minor $\mathrm{ABO} / \mathrm{RBC}$ mismatched transplantations induce PLS. It is possible that transferred donor B-lymphocytes were stimulated (an infection, for example) and/or recipient T-lymphocytes were abrogated (specific immunosuppression, such ATG) in order to allow donor $\mathrm{B}$ cell activation, maximizing donor blood group $\mathrm{Ab}$ production [2]. It has been suggested that a short cold ischemia time and a fast implantation process also favors PLS, due to the greater number and viability of the donor's lymphocytes [10].

Generally, this condition is mild and self-limited. Hemolysis stops with the decline of $\mathrm{Ab}$ production, due to the inability of renewal of donor B-lymphocytes or the development of tolerance of graft lymphoid tissue towards the recipient Ag. The 99 reported cases reviewed by Nadarajah L, et al. [1] showed a median time for hemolysis onset of 17 days (minimum of 5 days post-transplant, maximum 3 months after) and a duration of hemolysis from 3 to 183 days, with only 2 reported cases during more than 170 days. In our case, the patient developed AIHA before day 14 post-transplant, and showed evidence of hemolysis resolution at day 45.

The main treatment of PLS is supportive, with blood transfusions if necessary. RBC units of the donor blood group should always be used, as transfusion with recipient blood group RBC may exacerbate hemolysis. In our case, the patient was initially transfused with 8 units of $\mathrm{ORh}^{+}$, which may have perpetuated AHAI. Ramsey G [6] analyzed 18 kidney transplant recipients with PLS, reporting a median number of RBC units transfused of 6.5 (range 1 to 18 ).

The use of epoetin beta must be discontinued since it stimulates the recipient's erythropoiesis to produce recipient's RBC that will also perpetuate the mechanism, resulting in their destruction by donor's $\mathrm{RBC} \mathrm{Ab}$ in circulation and therefore prolonging the anemia.

The immunosuppression can also be increased [1-2,8], mostly mycophenolate mofetil (for its suppression effect on B cells) and corticosteroids. A high dose of corticosteroids is generally used, although without great evidence of efficacy; nonetheless, the potential benefit for a short-term period overlaps the eventual toxicity. We applied high dose corticosteroids in our patient for 14 days, a decision that was based on the clinical course observed, with progressive dose reduction afterwards.

Plasmapheresis and rituximab have also been suggested for management of severe cases of PLS, with a successful report on a PLS case occurred after small bowel transplantation [11]. However, a case of PLS in ABO-incompatible kidney transplant [12], after pretransplant desensitization protocol with rituximab and plasmapheresis was recently described, reinforcing the idea that the donor's transferred plasma cells, not affected by rituximab, are possibly the major PLS culprit.
Alloimmunization of the donor to the $\mathrm{Rh}(\mathrm{D}) \mathrm{Ag}$, as noticed in our case report, is a risk factor for PLS. Rh- women who had transplacental fetomaternal bleeding during any pregnancy of a $\mathrm{Rh}^{+}$fetus or who are otherwise exposed to $\mathrm{Rh}^{+} \mathrm{RBC}$ are at risk of developing anti-D Ab. Nevertheless, routine screening of the donors or risk stratification of recipients cannot be proposed as having an acceptable risk-benefit ratio, given the rarity of the condition [7].

Moreover, kidney transplantation can be securely performed in the presence of a minor $\mathrm{ABO} /$ other $\mathrm{RBC}$ mismatch, giving its selflimiting course and since long term graft function does not seem to be affected $[1-4,7,10]$, but the clinicians must be aware to the possibility of hemolysis PLS-induced and start support treatment as soon as detected. In our patient's case, the condition did not compromise the graft's viability and function, during the follow-up period available.

\section{Disclosure of Potential Conflicts of Interest}

None declared.

\section{References}

1. Nadarajah L, Ashman N, Thuraisingham R, Barber C, Allard S, et al. (2013) Literature review of passenger lymphocyte syndrome following renal transplantation and two case reports. Am J Transplant 13: 1594-1600.

2. Ainsworth CD, Crowther MA, Treleaven D, Evanovitch D, Webert KE, et al. (2009) Severe hemolytic anemia post-renal transplantation produced by donor anti-D passenger lymphocytes: case report and literature review. Transfus Med Rev 23: 155-159.

3. Elansary M, Hanna MO, Saadi G, ElShazly M, Fadel FI, et al. (2015) Passenger lymphocyte syndrome in $A B O$ and Rhesus D minor mismatched liver and kidney transplantation: A prospective analysis. Hum Immunol 76: 447-452.

4. Li FK, Chan TM, Lai KN (2000) Alloimmune hemolysis after renal transplantation. Am J Nephrol 20: 473-475.

5. Audet M, Panaro F, Piardi T, Huang P, Cag M, et al. (2008) Passenger lymphocyte syndrome and liver transplantation. Clin Dev Immunol 715769.

6. Ramsey G (1991) Red cell antibodies arising from solid organ transplants. Transfusion 31: 76-86.

7. Karanth P, Birchall J, Day S, Unsworth DJ, Ravanan R (2014) Immune hemolysis resulting from passenger lymphocyte syndrome derived anti-rh (D) reactivity after kidney transplantation: a case report and literature review. Transplantation 97: e54-e55.

8. Pomper GJ, Joseph RA, Hartmann EL, Rohr MS, Adams PL, et al. (2005) Massive immune hemolysis caused by anti-D after dual kidney transplantation. Am J Transplant 5: 2586-2589.

9. Antunes MB, Malheiro J, Dias L, Martins LS, Pedroso S, et al. (2019) Severe passenger lymphocyte syndrome associated to Rh antibodies after donor living renal transplantation. Port J Nephrol Hypert 33: 115-117.

10. Pascual-Pérez V, Gómez-Larrambe N, Collantes-Mateos R, VisusFernández de Manzanos T, Urbizu-Gallardo JM, et al. (2013) Passenger lymphocyte syndrome: an uncommon form of anaemia in renal transplantation. Nefrologia 33: 615-616.

11. Panaro F, DeChristopher PJ, Rondelli D, Testa G, Sankary H, et al. (2004) Severe hemolytic anemia due to passenger lymphocytes after living-related bowel transplant. Clin Transplant 18: 332-335.

12. Nishide S, Uchida J, Kabei K, Iwai T, Kuwabara N, et al. (2019) Passenger Lymphocyte Syndrome in the ABO-Incompatible Kidney Transplant Recipient Receiving Rituximab. Exp Clin Transplant 17: 558-560. 